\title{
A Review of Dementia Screening Tools based on Mobile Application
}

\author{
Fadi Thabtah \\ Digital Technologies, Manukau \\ Institute of Technology \\ Manukau, Auckland, New \\ Zealand \\ Fadi.fayez@manukau.ac.nz
}

\author{
David Peebles \\ Psychology department, \\ University of Huddersfield \\ Huddersfield, HD1 3DH, UK \\ dpeebles@hud.ac.uk
}

\author{
Jenny Retzler \\ Psychology department, \\ University of Huddersfield \\ Huddersfield, HD1 3DH, UK \\ jretzler@hud.ac.uk
}

\author{
Chanchala Hathurusingha \\ Digital Technologies, \\ Manukau Institute of \\ Technology \\ Manukau, Auckland, New \\ Zealand \\ Chanchala. Hathurusingha \\ @manukaumail.com
}

\begin{abstract}
Dementia is an umbrella term for multiple neurocognitive conditions that affect one's functional independence making daily activities challenging. Early screening can be critical for successful management of the disease, thus tools implemented within mobile technology to speed up accessibility are becoming more common. This research critically analyses dementia and mild cognitive impartment $(\mathrm{MCl})$ screening tools in terms of their clinical validity \& acceptability, efficiency, and performance. We used inclusion and exclusion criteria to search Android and Apple mobile stores, for dementia and $\mathrm{MCl}$ screening applications. The comparison of the prevailing apps suggests that no app fulfils the considered evaluation criteria. Hence, the development of dementia screening apps that cover cognitive, memory, and execution domains defined within the neurocognitive disorders for dementia diagnosis of Statistical Manual of Mental Disorders (DSM-5), may provide clear clinical benefit as these domains are crucial. Apps such as Cognity are promising as they include new intelligent features by using artificial intelligence to screen individuals.
\end{abstract}

\section{Keywords}

Accessibility, Dementia, Health Technology, Medical Screening, Medical Informatics, Mobile Application

Corresponding Author Details

Fadi Thabtah

School of Digital Technologies, Manukau Institute of Technology

Private Bag 94006, Manukau, Auckland, 2241, New Zealand.

E-mail: fadi.fayez@manukau.ac.nz.

\section{Declarations}

Funding: This study was not based on funding

Conflict of Interest: The authors declare that they have no conflict of interest.

Ethical Approval: This study involves no direct contact with human or animal and therefore no ethical approval is required. 


\title{
A Review of Dementia Screening Tools based on Mobile Application
}

\begin{abstract}
Dementia is an umbrella term for multiple neurocognitive conditions that affect one's functional independence making daily activities challenging. Early screening can be critical for successful management of the disease, thus tools implemented within mobile technology to speed up accessibility are becoming more common. This research critically analyses dementia and mild cognitive impartment (MCl) screening tools in terms of their clinical validity \& acceptability, efficiency, and performance. We used inclusion and exclusion criteria to search Android and Apple mobile stores, for dementia and $\mathrm{MCl}$ screening applications. The comparison of the prevailing apps suggests that no app fulfils the considered evaluation criteria. Hence, the development of dementia screening apps that cover cognitive, memory, and execution domains defined within the neurocognitive disorders for dementia diagnosis of Statistical Manual of Mental Disorders (DSM-5), may provide clear clinical benefit as these domains are crucial. Apps such as Cognity are promising as they include new intelligent features by using artificial intelligence to screen individuals.
\end{abstract}

Keywords: Accessibility, Dementia, Information Technology, Medical Screening, Medical Informatics, Mobile Application

\section{Introduction}

Dementia is the descriptive term used to define difficulties pertaining to an individual's memory, emotions, and cognitive and communication ability in performing normal day-to-day activities (Ernst \& Hay, 1999). A number of neurological diseases are categorised under the umbrella of dementia, including Alzheimer's disease (AD), Parkinson's disease, and Huntington's (Chertkow, 2008). The number of Americans diagnosed with dementia was estimated at 5.8 million in 2019, and of those, 5.6 million were aged 65 years or above. It is also reported that these numbers continue to increase annually with a growth rate of $3 \%$ among Americans aged $65-74,17 \%$ for people aged 75-84, and 32\% for people aged above 85 (Alzheimer's Association, 2019). Severe dementia can lead to profound consequences such as immobility and malnutrition, and eventually death. In 2019 there were 121,404 deaths from AD or other types of dementia reported in America (Alzheimer's Association, 2019). Although the risk of dementia increases with age, it is not a normal part of getting old. To reduce the impact of the severe consequences associated with dementia, it is imperative to identify any symptoms as early as possible, to allow for appropriate monitoring and intervention. Fortunately, the emerging use of technological advancements and mobile-based solutions has the potential to simplify this process for both the patient and clinician.

Today's healthcare industry embraces technological innovations to provide better personalised healthcare services to the public. Mobile-based solutions and applications' technology is one such revolutionary and trending innovation that facilitates enhanced patient care management and efficient diagnosis. The role of this technology in the healthcare industry is enormous as it improves the communication and integration of the entire healthcare process; this in turn enhances the productivity of the healthcare service providers and the healthcare system (Ventola, 2014).

According to statistics from the International Telecommunication Union (ITU) (2015), there were over 7 billion mobile phone subscriptions globally in 2014 , with $70 \%$ from low or middle-income developing countries. Ubiquity of mobile phone ownership provides an opportunity for alternative access to healthcare. A study by Wallace, Clark, and White (2012) showed that $87 \%$ of the physicians in the Manhattan Research Channel used smartphones or tablets in their work, and of those, $54 \%$ used mobile phones to support their practice and diagnosis. Mobile apps have become increasingly popular among the professionals and individuals involved in the healthcare sector, with a variety of Mhealth applications that have been developed to improve healthcare facilities for all kinds of patients.

Mobile application technology plays a vital role in screening, diagnosing, and treating cognitive impairments such as dementia. Screening that leads to early detection of cognitive dysfunction is important as it allows the individuals and their caregivers to access medical care services to design and implement treatment plans. In 
addition, the value of early detection using psychological tests allows clinical monitoring of disease progression (Panegyres, Berry, \& Burchell, 2016). Typically, existing mobile dementia screening tools provide a quick evaluation of different cognitive and functional aspects. Depending on the scores obtained from the test, these methods can pinpoint an individual's dementia traits and possible impairments.

Several research studies have been carried out to evaluate traditional dementia screening and diagnosis tools, i.e. (Snyderman \& Rovner, 2009; Kansagara \& Freeman, 2010; Newman et al., 2018; Bruno \& Vignaga, 2019 ); however, to date few research studies have focused on evaluating mobile application screening tools (Yoganathan 2013 ; Berauk et al., 2017). This is required not only to help clinicians and healthcare professionals to determine the suitable dementia screening tools to support their medical diagnosis, but may also be of use to potential patients and caregivers. Therefore, the current review aims to fill the gap of previous reviews and critical analysis research related to dementia screening, by identifying $\mathrm{MCl} \&$ dementia screening tools available on both Apple and Android platforms. This paper reviews existing mobile apps for dementia, summarising each based on their ratings, number of downloads, feedback, performance (sensitivity and specificity), and usability (if based on a medically accepted examination). More specifically, we investigate features including number of items, the use of audio and video technology, supported languages, accessibility (presence on both Android and iOS platforms/target audience/ cost), comprehensiveness (number of cognitive areas covered), clinical validity, and acceptability (use of clinically validated medical examination method), efficiency (time to complete the test), performance (sensitivity, specificity), user rating, cost, feedback, and number of downloads among others. This will help clinicians, patients, and caregivers identify valid mobile apps that they can use to screen patients for dementia or $\mathrm{MCl}$.

The paper consists of five main sections. Section Two reviews and evaluates dementia screening tools available in the mobile application environment. Section Three critically analyses each instrument based on clinical predetermined criteria and Section Four concludes the findings and suggests future study.

\section{Literature Review}

There are a number of mobile apps available in the Apple and Google stores to assist individuals who are already diagnosed with dementia and $\mathrm{MCls}$. Apple and Android app stores were searched for apps that met the following inclusion criteria:

1) Tools that only consider screening of dementia, $A D$ and $M C I$

2) Tools that are available in English

3) Tools that are based on valid medical tests

All other apps related to $\mathrm{MCl}, \mathrm{AD}$ and dementia that do not fulfill one or more of the above inclusion criteria were excluded from this research. Only 20 screening apps have been retained using the inclusion criteria. Discussed below are apps available for screening dementia and $\mathrm{MCl}$ that can be utilised to assist healthcare professionals in their medical diagnosis.

\subsection{Cognitive and Memory Apps}

\section{BrainCheck}

BrainCheck was launched to screen individuals with cognitive dysfunction during routine medical examination and is only available in the Apple store (Ehrensperger, et al., 2014). This partially self-administered tool consists of various tasks for the patient (Holsinger, Deveau, Boustani, \& William, 2007), involving a Clock Drawing Test (CDT) (Friedman, et al., 1994), and seven questions derived from the 7-item version of the Informant Questionnaire on Cognitive Decline in the Elderly (IQCODE) (Jorm, Scott, \& Jacomb, 1989); it takes ten minutes to complete. The BrainCheck app doesn't generate a score, but it allows the users to share the test results with a healthcare professional to support the diagnosis. According to Ehrensperger et al., (2014), BrainCheck has a sensitivity of $97.4 \%$ and specificity of $81.6 \%$ in identifying cases of dementia and $\mathrm{MCl}$. 


\section{Brain Check Inc.}

BrainCheck Inc (BCl) (BrainCheck, 2019) has five different games developed based on gold-standard neurocognitive tests: the Flanker Task (Fan, Flombaumb, McCandlissa, Thomas, \& Posner, 2003), the Digit Symbol Substitution Task (Monte, Geffen, May, \& McFarland, 2010), the Stroop Task (Melara \& Algom, 2003), the Trail Making Test (TMT) (Cicerone \& Azulay, 2002), balance and coordination (Greenwald, et al., 2001), and the Immediate and Delayed Recall Tests (Comerford, Geffen, May, Medland, \& Geffen, 2002). Based on the results of each game, the app presents a graph that indicates the level of cognitive function pertaining to cognitive processing, executive function, visual attention, immediate recall, and delayed recall abilities of the user. $\mathrm{BCl}$ is designed to be administered by a clinician and healthcare professional and registered as a Class II medical device with the U.S. Food and Drug Administration (FDA); it has a sensitivity of $83 \%$ and specificity of $87 \%$ (Yang, et al., 2017).

\section{MMSE}

The MMSE app (Google, 2016) is the mobile digitalized version of the Mini Mental State Examination (MMSE) test (Folstein, et al, 1975). On completion of the questionnaire, the app generates a score out of 30 based on the provided answers. It also allows the user to share and monitor the test results and set daily reminders to do the test. It has gained a 3+ rating on Google Play (Klimova, 2017). The Dementia \& Alzheimer's Memory Diagnosis Test (DAMDT) (Google, 2017) is another mobile version of MMSE. It helps the users to assess their cognitive health and recommends seeking further medical advice and support if necessary. The questionnaire mainly tests the memory capacity and orientation of the individual and then generates a score out of 30 . Anyone receiving a score below 25 is recommended to seek further medical assistance. DAMDT Test app version of the MMSE gained 3.4/5 rating on Google IPay with over 5000 downloads and is freely available.

\section{Brain Test}

BrainTest, (BrainTest Inc, 2013) was developed based on Self-Administered Gerocognitve Exam (e-SAGE) (Scharre et al., 2010) for the early detection AD and other associated MCl (Scharre, et al., 2010). BrainTest is available in both Apple and Google stores and can be administered by oneself or a caregiver and takes approximately 10-15 minutes to complete. According to Scharre, et al (2017), BrainTest has a sensitivity of $71 \%$ and specificity of $90 \%$, which is similar to the clinically used pen and paper-based counterparts such as the Montreal Cognitive Assessment (MoCA) (Nasreddine et al., 2005) and MMSE.

\section{Cognitive Exams}

Cognitive Exams (Google, 2019a) is a dementia screening tool developed for research purposes using interactive tools such as MMSE, Clock Test, Geriatric Depression Scale (Yesavage, et al., 1982), Katz Examination (Aske, 1990), Walk Test of 6 minutes (Abbott, et al., 2004), and a Fluency Test (Caramelli, Carthery-Goulart, Porto, Charchat-Fichman, \& Nitrini, 2007). The interactive activities available in this tool assess cognitive (memory, verbal fluency, depression, etc.), functional (bathing, dressing, toileting, transferring, continence, and feeding), and cardiopulmonary health areas of the suspected individual. Cognitive Exams is an unconventional tool that takes approximately 45 minutes to complete and requires administration by a specialized professional in a formal clinical setting. The Cognitive Exams app has a 4.4 rating on Google Play and reported over 10,000 downloads.

\subsection{Unstructured Dementia Screening Apps}

\section{MOBI-COG}

MOBI-COG (Nirjon et al., 2014) is an app based on the traditional Mini-COG dementia medical exam (Borson, et al, 2000) to identify dementia in patients with cognitive impairments. The pilot version of the MOBI-COG was launched on the Android platform in 2014 and consists of a three-minute test that can be administered by either patient or a caregiver. The app is designed to measure the user's ability to recall words, digital positioning, digit values, and positioning of the hands of a clock using unique tests (Task 1: Remembering Words; Task 2: Clock drawing task; Task 3: Recalling words). It generates a score ranging from 1-10 to measure the cognitive function level of the user and a healthcare professional is required to interpret the results to make a formal diagnosis. The MOBI-COG app was well-trained using 1000 sample clock drawings to optimize the accuracy (99.53\%) of its outcome; according to Nirjon et al., (2014), this version of the MOBI-COG gained a 
rating of 8.5 out of 10 . Neither the pilot version nor the finalized version can be found in the Google or Apple stores.

\section{The CAIDE Dementia Risk Score App}

The CAIDE Dementia Risk Score (CAIDE-DRS) (Sindi et al., 2015) app is based on Cardiovascular Risk factors, Aging, and Incidents of Dementia risk score (CAIDE) (Kivipelto, et al., 2006). It was developed to assist users determine the risk of developing MCIS within 20 years, along with providing suggestions on modified actions, guidance, and recommendations. The app calculates the user's risk of developing dementia using five indices: Personal data (name, age, gender, and education), Systolic Blood Pressure (BP), Body Mass Index (weight and height $\left(\mathrm{kg} / \mathrm{M}^{2}\right)$ ), Cholesterol Level, and Physical Activity (high/low) of the user. This app generates a score out of 15 based on the answers provided with the cut-off score set at 9. The CAIDE-DRS app exhibited a sensitivity of $77 \%$ and specificity of $63 \%$ (Butcher, 2007).

\section{Dementia Risk Tool}

The design of the Dementia Risk tool (DRT) (Google, 2019b) is quite similar to the CAIDE-DRS app (Kivipelto, et al., 2006) and uses almost the same set of indices. The DRT was developed to self-assess the user's possibility of developing dementia or $A D$ and is freely available in both the Apple and Google stores. On completion of the questionnaire, a score between $0-15$ is given where $0-5$ indicates a risk of $1 \%, 6-7$ indicates a risk of $1.9 \%$, 8-9 indicates a risk of $4.2 \%, 10-11$ indicates a risk of $7.4 \%$, and $12-15$ indicates a risk of $16.4 \%$ of developing dementia or any other associated $\mathrm{MCl}$. The score presents a series of recommendations and corrective actions that the user can consider to further reduce risk. This screening tool has a rating of 4.8/ 5 on Google Store.

\section{eSLUMS}

eSLUMS (ChewyLogic, 2019) is based on the digital version of the Saint Louis University Mental Status (SLUMS) Exam (Tariq et al, 2006). eSLUMS is freely available in the Google Appstore and takes 7-10 minutes to complete. The Professional version of eSLUMS provides more advanced features including the ability to view the patient's entire medical history while managing multiple patients, then generating the test results as a complete softcopy report. eSLUMS is designed to be administered by medical professionals with automatic scoring that ranges from 0-30, where 27-30 indicates normal cognitive function, 21-26 indicates mild cognitive dysfunction, and a score below 20 indicates severe cognitive impairment. eSLUMS has a sensitivity and specificity range of $98 \%-100 \%$ respectively (Kansagara \& Freeman, 2010).

\section{Cognity - Alzheimer's and Dementia Test}

Cognity is available in both Google and Apple Stores (Inoven, 2018). This app utilizes the efficient Al method with the digitalized Mental Status Examination (MSE) tool (Snyderman \& Rovner, 2009) to compute the potential risk of Visuospatial Memory problems and dementia. This is one of the first screening apps to use Al to detect cognitive dysfunction through analysis of a clock drawing. It takes 3-5 minutes to identify Visuospatial Memory risk and approximately 10 minutes to detect dementia traits. It is also one of the few dementia screening apps that has pre-recorded videos that explain and interpret the final risk score (\%) based on answers given on 30-items of MSE and photo of a clock drawing. The app is available to download freely, but the dementia test can be purchased for US\$ 8.99. It is rated 3+ on the Apple Store, and sensitivity and specificity of the MSE medical examination ranges from $71-92 \%$ and $52-96 \%$ respectively (Snyderman and Rovner, 2009).

\section{Confusion: Delirium \& Dementia}

Confusion: Delirium \& Dementia's (CDD) (Sangha, George, Winthrop, \& Panchal, 2015) aim is to manage and ensure the safety of confused individuals who are suffering various complications associated with cognitive impairments. The CDD app is available on the Android platform for medical professionals and researchers. It is the only UK-NHS clinically accepted mobile app that assists general practitioners to distinguish between delirium and dementia in suspected individuals (Yoganathan, 2013). It uses the Confusion Assessment Method 
(CAM) (Inouye, et al., 1990), Six Item Screener (Callahan, Unverzagt, Hui, Perkins, \& Hendrie, 2002), Abbreviated Mental Test (AMT) (Hodkinson, 1972), MoCA, and MMSE to identify delirium and dementia. At the end of each assessment, CDD presents a score, from which the practitioner can determine delirium, dementia, and other cognitive impairments. Tips for prevention, information on suitable medication, and recommendations for treating individuals who are diagnosed with dementia or any other type of cognitive disorder can then be provided.

\section{Dementia Screener}

DementiaScreener (Mundt, Freed, \& Greist, 2000) was developed mainly for experimental purposes and can be administered either by a caregiver or the individual. DementiaScreener provides two screening assessments to estimate the risk of dementia. The first one is Symptoms of Dementia Screener (SDS) (Flaherty, Midden, \& Mast, 2019) which evaluate the dementia symptomology based on communication and behavioural characteristics such as repetition of questions, forgetfulness, need for reminders, mood swings, difficulties in calculation and problem solving, concerns in driving, etc. Based on the given answers, the tool presents a score which if $<4$ indicates lower likelihood, and a score $>4$ indicates higher likelihood of developing a cognitive impairment. The other type of screening mechanism provided by the app is the AD8 Dementia Screening Interview (Galvin, et al., 2005) where the behavioural changes are identified through a questionnaire answered by the caregiver (if available, otherwise the patient) to evaluate the final score, and thus determine the presence of cognitive impairment. A score of 0-1 reflects Normal Cognition whereas a score of 2 or greater suggest cognitive impairment is likely.

\section{ACE Mobile}

The ACE mobile (Hodges \& Larner, 2017) is based on the ACE-III (Noone, 2015). The ACE mobile is freely available on iTunes (only supports iPads). It is recommended that this tool be administered by a healthcare professional in a proper clinical setting. ACE mobile employs 24-items to evaluate five cognitive functional areas of the user: attention (18 points), memory (26 points), fluency (14 points), language (26 points), and visuospatial functioning (16 points), and then generates a total score out of 100 (Newman, et al., 2018). The tool has exhibited a high level of sensitivity $(93 \%-100 \%)$ and specificity $(96 \%-100 \%)$ when the cut-off score was set at 82-88 (Bruno \& Vignaga, 2019). This screening app has gained a 4+ rating on iTunes.

\section{Alzheimer's Disease Pocket Card}

The Alzheimer 's Disease Pocket Card (ALZ) (Berauk, et al., 2017) is a tool that provides educational materials, information on detection, and guidance on pharmacotherapy and non-pharmacologic strategies that help to manage AD. This app, is freely available on Apple iTunes. It uses the Mini-Cog Dementia Screening Test (Borson et al., 2000), Memory Impairment Screen (MIS) (Buschke, et al., 1999), Clock Drawing Test (Friedman et al., 1994), General Practitioner Assessment of Cognition (GPCOG) (Brodaty, et al., 2002), and the Montreal Cognitive Assessment (Nasreddine, et al., 2005) as cognitive assessment algorithms. Since ALZ is a patient administered tool, it also uses a 7-item version of the IQCODE (Jorm, Scott, \& Jacomb, 1989) and the AD8 Dementia Screening Interview (Galvin , et al., 2005) as informant administered tools, and SLUMS (Tariq et al., 2006), the Functional Activities Questionnaire (FAQ) (Tappen, Rosselli, \& Engstrom, 2009), the Geriatric Depression Scale (Yesavage, et al., 1982), the Hachinski Ischemic Score (HIS) (Johnson, et al., 2014) and the Katz basic activities of daily living as functional assessment tools to screen for AD. It also allows people to network with others who are having similar problems or difficulties and takes approximately 45 minutes to complete but doesn't have a sufficient number of ratings on the App Store.

\section{Dementia Test - risk calculator of dementia}

The Dementia Test - risk calculator of dementia (DTRCD) (Apple 2019b) is an iOS app developed to calculate the potential risk of an individual having dementia based on a simple self-administered questionnaire. This tool is free and, according to the developers' note on Apple iTunes, this app uses (Torre, 2004) a novel memory test based on a combination of multiple algorithms and a wide range of factors that affect the memory, attention, and logical thinking abilities of an individual to detect the risk of Dementia. It also provides disease information required to manage the disease, reminders on next test date, ability to share test results via e- 
mails, and recommendations and instructions to reduce the risk of dementia. Although the app has a rating of $5 / 5$ on the App Store, there is not much information available on its functionality or effectiveness.

\section{DementiaTest}

The DementiaTest (Thabtah, 2019) app was developed exclusively for research purposes. Hence it is freely available on both iTunes and Google Play to download and use and can be administered by caregivers. The app helps the users to identify symptoms of dementia through medical questionnaires based on the 6-item Cognitive Impairment Test (6-CIT) and Structured Clinical Interview (SCIDS) (Callahan et al.; Ouimette \& Klein, $1995)$ tests. The DementiaTest app has two separate questionnaires: a self-administered questionnaire, and a caregiver-administered questionnaire. Based on the answers given for each question, the self-administered questionnaire generates a score out of 26 , and the other a score out of 36 ; a lower score indicates normal cognitive behaviour, whereas a higher one is indicative of dementia traits. This app has gained a $3+$ rating on the App Store and takes no longer than five minutes to complete.

\section{MoCA APP}

The MoCA app (Apple, 2019c) is a screening and diagnosis tool for many conditions such as vascular dementia, Parkinson's disease, traumatic brain injury, brain tumour, Huntington's disease, etc. (Nasreddine et al., 2005). This tool is available in the App Store and can only be downloaded into an iPad. The MoCA app focuses on the abilities of executive functions, language, attention, memory recall, concentration, time and place awareness to evaluate an individual's vulnerability to different mental and execution areas. The tool uses a questionnaire consisting of 11 questions with various drawing tasks to identify possible cognitive impairments and takes no longer than 10 minutes to complete. On completion, a score out of 30 is generated where scores above 26 are considered as normal cognition. According to a study by Julayanont et al., (2015), the MoCA app screens MCl with $81 \%$ sensitivity and $86 \%$ specificity when the cut-off score is set around $24-25$. The app has rated $4+$ on iTunes.

\section{DST - Dementia Screening Test, Alzheimer Test}

The DST app (Google, 2019c) is based on the Dementia Screening test (DST) medical examination that has the ability to detect different types of dementia conditions including AD, Vascular dementia, Fronto-temporal dementia, Lewy-body dementia, and Secondary dementia along with their severity levels. It is available on both iOS and Android platforms. According to the developer's note, the app has a sensitivity of $96 \%$. The user is allowed to access the screening test without registration, storage of personal data, advertisements, and even without internet access. Clock drawing is one of the tasks that this tool employs to assess the executive functions and memory skills of the user to detect their potential for developing dementia. The DST takes 10-15 minutes to complete. The app generates a score out of 30 based on the answers given to test items; scores 2930 is considered as normal, score between $24-28$ is considered as having slight limitations in some areas and score below 23 indicates limitations are present. It has gained 3+ rating with over 100 downloads on the App Store.

\section{Cognitive Item Test (6CIT) App}

The 6CIT app (Apple, 2019a) is a MCl screening tool based on the Six Cognitive Item Test (Callahan et al., 2002). The app consists of 6-items and generates a score out of 28. Test scores from 0-7 indicate normal cognition and scores from 8-28 indicate an abnormality in cognition and suggest further evaluation for diagnosis. The study by Callahan et al., (2002) validated the reliability of the app with a sensitivity ranges from $78-90 \%$ and specificity of $100 \%$ when the cut-off score is $7-8$. This app is freely available I nthe Apple Store and takes five minutes to complete. However, this app does not have enough ratings for the App Store.

\section{Discussion}

Table 1 presents an overview of the selected dementia screening apps based on target audience, ratings on the App Store, number of downloads, availability to download freely, types and number of questions used, average time taken to complete the activities, cost, and number of languages supported by the app. Accessibility is a vital measure that describes the suitability and acceptability of a dementia screening app. This 
paper evaluates the accessibility of considered dementia screening apps based on their presence in both Android and iOS platforms, audience covered, and cost.

The CDD app and ACE-Mobile are designed exclusively for healthcare professionals and orthopaedic trauma patients respectively, restricting their accessibility. Other apps, such as the MOBI-COG, DementiaScreener, Brain Test, MoCA, eSLUMS, DST, and MMSE can be used by anyone to screen for dementia. According to Statista, (2018), the global mobile operation systems (OS) market has $88 \%$ of Andriod OS shares and $11.9 \%$ of iOS; having a presence in both these platforms, makes these apps more accessible. Out of all the apps considered, BrainTest, ACE, DRT, Cognity, DST, and DementiaTest are the ones that are presented in both platforms, making them more accessible than the other apps.

Similarly, some apps are not freely available to users (ex: ACE-Mobile). BrainTest, ACE Mobile, DRT, Cognity, and DementiaTest are available on both Android and Apple platforms making them more accessible to the public and health professionals. Although ACE-Mobile is available for free to download, it only supports iPads and tablets and is designed to be used by clinicians. In terms of the time taken to complete the assessment, MOBI-COG and DRT take only 2-3 minutes to complete, however, they do not cover all neurocognitive areas related to dementia diagnosis. Considering multimedia features, only the BrainTest and DST apps utilize videos and images making them attractive.

Screening apps such as ALZ and Cognitive Exams contain over 50 items (100+ and 68 respectively) and take 45 60 minutes to complete. These two apps are suitable for clinical set up examination since they are administered by a clinician and contain multiple valid medical tests. In contrast, the MOBI-COG, BCl and $6 \mathrm{CIT}$ apps only have 5-6 items and take no longer than five minutes to complete. Apps like Cognity and DST use video and images to present the items making them more appealing to users. These apps take 10-15 minutes to complete which is reasonable. Most of the considered dementia screening apps have obtained 4+ ratings on the App Store. The MOBI-COG, BrainTest, and DRT apps reported the highest ratings of 8.5/10, 4.5/5, and $4.8 / 5$ respectively; the DRT app reported over $1000+$ downloads making it the most popular.

Table 2 presents a comparison of the considered dementia screening apps using the cognitive domain measured, medical assessment used, and performance. In terms of the medical exams used, almost all the considered apps are designed on common, clinically accepted tests such as MMSE, MoCA, Mini-COG dementia screening test, AD8 Dementia Screening Interview, 7-item version of the IQCODE and GPCO - all validated by the Alzheimer's Association as clinically accepted tools for screening dementia (Cordella, et al., 2013). The best performing medical tools are those with the best trade-off between sensitivity and specificity values. The DementiaTest, ACE, MOBI-COG and e-SLUMS consist of medical tests that appear to have a good trade-off between sensitivity and specificity values. Comprehensiveness is another measure that evaluates the usability and functional capabilities of these apps for identifying impairments pertaining to various cognitive domains. All the considered apps cover a wide range of cognitive and functional domains such as attention, memory, language, and visuospatial ability. Almost all of the apps focus on memory. Apps such as ACE-Mobile, CognitiveExams, DST, ALZ, MoCA, and BCI are more comprehensive than the other apps covering multiple cognitive and executive domains. Apps such as the CAIDE-DRS and DRT also cover hypertension, hypercholesterolemia, obesity, and physical inactivity.

The American Psychiatric Association (2013) in DSM-5 describes the criteria for diagnosing possible and probable AD. First some level of dementia or neurocognitive disorder must be established. Major neurocognitive disorder (major ND) requires the patients experience a significant decline over time in at least one of the following six cognitive domains: complex attention, executive function, learning and memory, language, perceptual-motor, or social cognition. This can be reported either by the patient, an informant or the clinician and then corroborated by performing a cognitive test that is associated with the domain. Alternatively, minor neurocognitive disorder (minor ND) requires a moderate decline in cognitive domains over time and that the patient's independence during everyday activities is not affected. Both major ND and minor ND also require that the cognitive defects are not only observed when the patient is delirious, and the cognitive defects are not better explained by another mental disorder.

Most of the screening apps that we reviewed cover one or more cognitive domains related to DSM-5. It is clear that some apps cover multiple neuropsychological domains based on a medical instrument but take more time to complete (ex: Cognitive Exams). Other apps utilize all the items of the corresponding medical assessment method while others only use a few items presented in the original medical assessment. Apps like MoCA covers activities related to social cognition, complex attention, learning \& memory, language and perceptual motor skills, and ALZ app has over 100 items and activities combining seven different medical tests, making 
them suitable for clinical use. Therefore, once major ND or minor ND has been demonstrated using some of the screening apps discussed, the DSM- 5 criteria can be used to determine if the observed disorder is being caused by AD.

Table 1: Dementia Screening Mobile Applications Overview

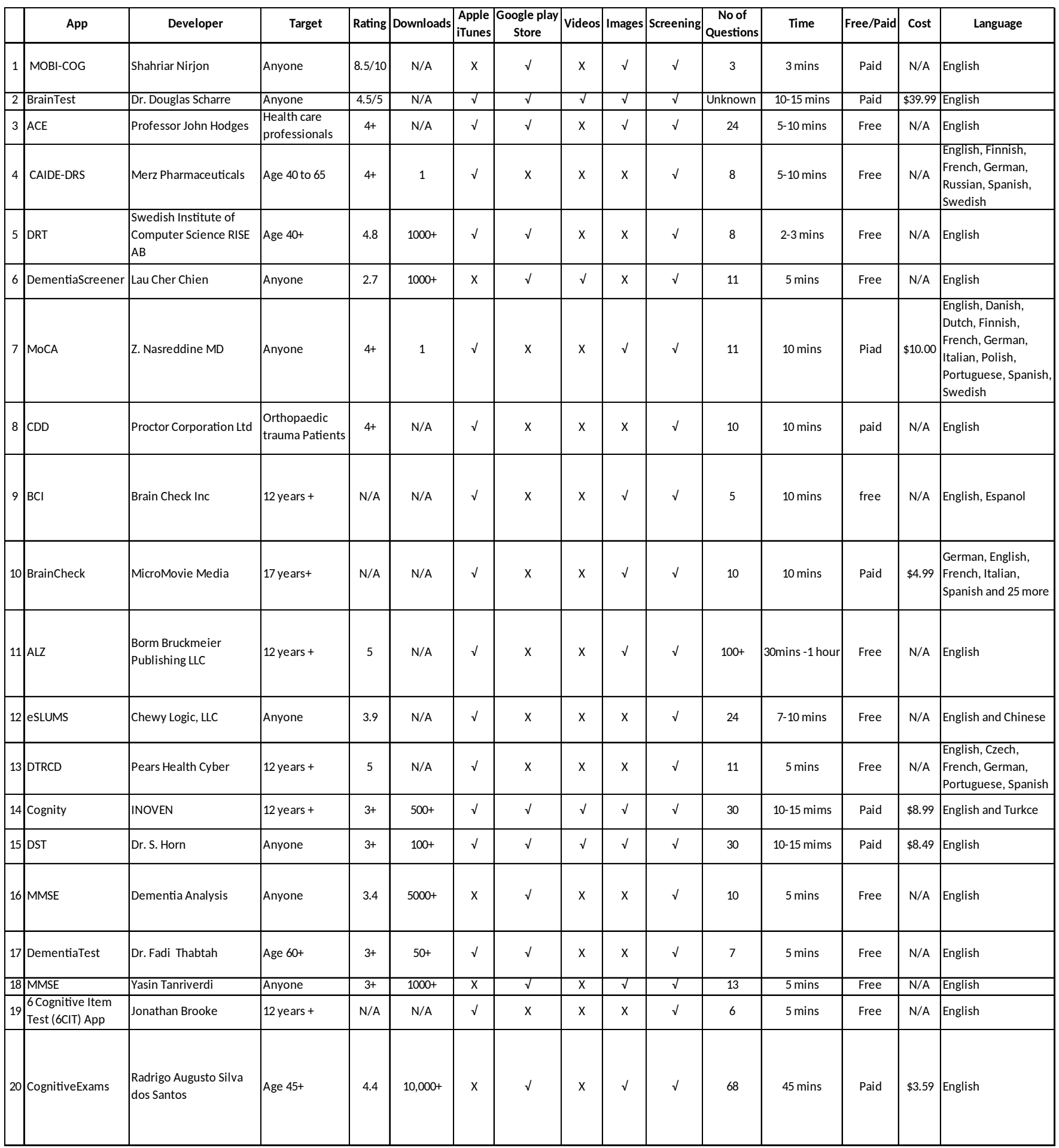


Table 2: Medical Examination Method, Performance, and Neurocognitive Areas the Apps Cover

\begin{tabular}{|c|c|c|c|c|c|c|}
\hline App & Areas Covered & Medical examination & Medical exam reference & Sensitivity & Specificity & Study performance reference \\
\hline MOBI-COG & Memory & Mini-COG dementia screening test & $\begin{array}{l}\text { (Borson, Scanlan, Brush, Vitaliano, \& } \\
\text { Dokmak, 2000) }\end{array}$ & $95.00-100.00 \%$ & $92.00-98.00 \%$ & $\begin{array}{l}\text { (Borson S., Scanlan, Watanabe, Tu , \& } \\
\text { Lessig , 2006) }\end{array}$ \\
\hline BrainTest & Memory and thinking & Self-Administrated Gerocognitve Exam (e-SAGE) & (Scharre D., et al., 2010) & $71.00 \%$ & $90.00 \%$ & $\begin{array}{l}\text { (Scharre D. , Chang, Nagaraja, Vrettos, } \\
\& \text { Bornstein, 2017) }\end{array}$ \\
\hline ACE & $\begin{array}{l}\text { Attention, memory, fluency, language, and } \\
\text { visuospatial functioning }\end{array}$ & Addenbrooke's Cognitive Examination (ACE-III) & (Noone, 2015) & $93.00-100.00 \%$ & $96.00-100.00 \%$ & (Bruno \& Vignaga, 2019) \\
\hline CAIDE-DRS & \begin{tabular}{|l|} 
Age, educational level, hypertension, \\
hypercholesterolemia, obesity, and physical \\
inactivity
\end{tabular} & $\begin{array}{l}\text { Cardiovascular Risk factors, Aging and Incidents } \\
\text { of Dementia risk score(CAIDE) risk score }\end{array}$ & (Kivipelto, et al., 2006) & $77.00 \%$ & $63.00 \%$ & (Butcher, 2007) \\
\hline \multirow{2}{*}{ DementiaScreener } & \multirow{2}{*}{$\begin{array}{l}\text { Decision making, memory, thinking, language } \\
\text { and behaviour }\end{array}$} & Symptoms of Dementia Screener (SDS) & (Flaherty, Midden, \& Mast, 2019) & $78.40 \%$ & $84.00 \%$ & (Flaherty, Midden, \& Mast, 2019) \\
\hline & & AD8 Dementia Screening Interview & (Galvin, et al., 2005) & & & \\
\hline MoCA & $\begin{array}{l}\text { Executive functions, language, attention, } \\
\text { memory recall, concentration and time and } \\
\text { place awareness }\end{array}$ & Montreal cognitive assessment & (Ziad S. Nasreddine, et al., 2005) & $81.00 \%$ & $86.00 \%$ & (Julayanont, et al., 2015) \\
\hline \multirow{3}{*}{ CDD } & \multirow{3}{*}{$\begin{array}{l}\text { Short and long term memory, attention and } \\
\text { orientation }\end{array}$} & Confusion assesment method (CAM) & ( Inouye, et al., 1990) & $94.00-100.00 \%$ & $89.00-95.00 \%$ & (Waszynsk, 2012) \\
\hline & & Six item screener & $\begin{array}{l}\text { (Callahan, Unverzagt, Hui, Perkins, \& } \\
\text { Hendrie, 2002) }\end{array}$ & $68.00-80.00 \%$ & $74.00-80.00 \%$ & $\begin{array}{l}\text { (Carpenter, DesPain, Keeling, Shah, \& } \\
\text { Rothenberger, 2011) }\end{array}$ \\
\hline & & Abbriviated mental test (AMT) & (Hodkinson H. , 1972) & $70.00-80.00 \%$ & $74.00-90.00 \%$ & (Jitapunkul, Pillay, \& Ebrahim, 1991) \\
\hline \multirow{6}{*}{$\mathrm{BCl}$} & \multirow{6}{*}{$\begin{array}{l}\text { Cognitive processing, executive Functions, } \\
\text { visual attension, immediate recall and } \\
\text { delayed recall (memory) }\end{array}$} & Flanker Task & $\begin{array}{l}\text { (Fan, Flombaumb, McCandlissa, Thomas, \& } \\
\text { Michael I, 2003) }\end{array}$ & \multirow{6}{*}{$81.00 \%$} & \multirow{6}{*}{$94.00 \%$} & \multirow{6}{*}{ (Groppell, et al., 2019) } \\
\hline & & The Digit Symbol Substitution Task & (Monte, Geffen, May, \& McFarland, 2010) & & & \\
\hline & & The Stroop Task & (Melara, \& Algom, 2003) & & & \\
\hline & & The Trail Making Test (TMT) & (Cicerone \& Azulay, 2002) & & & \\
\hline & & Balance and coordination & (Greenwald, et al., 2001) & & & \\
\hline & & The Immediate and Delayed Recall Tests & $\begin{array}{l}\text { (Comerford, Geffen, May, Medland, \& } \\
\text { Geffen, 2002) }\end{array}$ & & & \\
\hline \multirow{3}{*}{ BrainCheck } & \multirow{3}{*}{ Memory } & 3 patient questions (BrainCheck) & $\begin{array}{l}\text { (Holsinger, Deveau , Boustani , \& Williams, } \\
2007)\end{array}$ & \multirow[t]{2}{*}{$85.80 \%$} & \multirow[t]{2}{*}{$74.30 \%$} & \multirow{3}{*}{ Ehrensperger, et al., (2014) } \\
\hline & & Clock drawing test (CDT) & (Friedman , et al., 1994) & & & \\
\hline & & $\begin{array}{l}\text { 7-item version of the Informant Questionnaire on } \\
\text { Cognitive Decline in the Elderly (IQCODE) }\end{array}$ & (Jorm, Scott , \& Jacomb, 1989) & $81.40 \%$ & $75.70 \%$ & \\
\hline \multirow{4}{*}{ ALZ } & \multirow{4}{*}{$\begin{array}{l}\text { Interactive Skills, memory, mental health, } \\
\text { emotions, hypertension, atheroscierosis \& } \\
\text { etc }\end{array}$} & Functional Activities Questionnaire (FAQ) & (Tappen, Rosselli, \& Engstrom, 2009), & $80.30 \%$ & $87.00 \%$ & (Teng, et al., 2011) \\
\hline & & Hachinski Ischemic Score (HIS) & (Johnson, et al., 2014), & $89.00 \%$ & $89.30 \%$ & (Moroney, et al., 1997) \\
\hline & & Memory Impairment Screen (MIS) & (Buschke, et al., 1999) & $68.00-86.00 \%$ & $84.00-96.00 \%$ & (Tsoi, Chan, Hirai, Wong, \& Kwok, 2015) \\
\hline & & $\begin{array}{l}\text { General practitioner assessment of cognition } \\
\text { (GPCOG) }\end{array}$ & (Brodaty, et al., 2002) & $85.00 \%$ & $86.00 \%$ & (Brodaty, et al., 2002) \\
\hline esLUMS & Memory, attention, logical thinking & $\begin{array}{l}\text { Saint Louis University Mental Status (SLUMS) } \\
\text { Exam }\end{array}$ & Saint Louis University Mental Status (SLUMS) & $98.00-100.00 \%$ & $98.00-100.00 \%$ & (Kansagara \& Freeman , 2010) \\
\hline Cognity & Visuospatial, memory & Mental Status Examination (MSE) tool & (Snyderman \& Rovner, 2009) & $71.00-92.00 \%$ & $52.00-96.00 \%$ & (Snyderman \& Rovner, 2009) \\
\hline DST & \begin{tabular}{|l|} 
Executive functions, memory, verbal fluency, \\
attention and orientation
\end{tabular} & Dementia Screening Test & Dr. S. Horn (2019) & $96.00 \%$ & N/A & Developer's note on Appstore \\
\hline MMSE & Memory and attention & Mini Mental State Examination (MMSE) & (Folstein, Folstein, \& McHugh, 1975) & $23.00-76.00 \%$ & $40.00-94.00 \%$ & (Arevalo-Rodriguez, et al., 2015) \\
\hline \multirow{2}{*}{ DementiaTest } & \multirow{2}{*}{ Functional and cognitive areas } & 6-item Cognitive Impairment Test (6CIT) & $\begin{array}{l}\text { (Callahan, Unverzagt, Hui, Perkins, \& } \\
\text { Hendrie, 2002) }\end{array}$ & $78.00-90.00 \%$ & $100 \%$ & $\begin{array}{l}\text { (Callahan, Unverzagt, Hui, Perkins, \& } \\
\text { Hendrie, 2002) }\end{array}$ \\
\hline & & Structured Clinical Interview (SCIDS) & (Ouimette \& Klein, 1995) & $75.00-100.00 \%$ & $100 \%$ & $\begin{array}{l}\text { (Gerdner , Kestenberg, \& Edvinsson, } \\
\text { 2014) }\end{array}$ \\
\hline \multirow{4}{*}{ CognitiveExams } & \multirow{4}{*}{$\begin{array}{l}\text { Memory, verbal fluency, mental health, } \\
\text { depression, functional performance in } \\
\text { bathing, dressing, toileting, transfering, } \\
\text { continence and feeding and } \\
\text { caridiopulmonary health }\end{array}$} & Geriatric Depression Scale & (Yesavage, et al., 1982) & $92.50 \%$ & $77.20 \%$ & $\begin{array}{l}\text { (Mitchell, Bird, Rizzo, \& Meader, } \\
\text { 2011) }\end{array}$ \\
\hline & & $\begin{array}{l}\text { Katz basic activities of daily living as functional } \\
\text { assessment }\end{array}$ & (Aske, 1990) & $38.00 \%$ & N/A & (Hartigan, 2006) \\
\hline & & Walk Test of 6 minutes & ( Abbott, et al., 2004) & $82.00 \%$ & $84.00 \%$ & (AbilityLab, 2019) \\
\hline & & Fluency Test & $\begin{array}{l}\text { (Caramelli, Carthery-Goulart, Porto, } \\
\text { Charchat-Fichman, \& Nitrini, 2007). }\end{array}$ & $\mathrm{N} / \mathrm{A}$ & $\mathrm{N} / \mathrm{A}$ & N/A \\
\hline
\end{tabular}

Artificial intelligence (AI) techniques particularly deep learning and machine learning (ML) offer powerful capabilities for computer aided diagnosis (CAD) systems including those related to neurocognitive conditions (Chua, et al., 2019; Thabtah et al., 2019; Thabtah, 2019). These techniques explore historical data to derive useful patterns and concealed knowledge that can be utilized by clinicians and physicians to improve the accuracy of the diagnosis process. The potential use of $\mathrm{Al}$ and $\mathrm{ML}$ techniques in CAD systems related to dementia is vast, nevertheless few applied research studies have been done to investigate how to integrate Al 
technology with traditional diagnosis procedures, i.e. (Thabtah et al., 2020; Baadel et al., 2020; Chua, et al., 2019; Newman et al., 2018). One of the distinctive advantages that Al techniques offer besides improved predictions is the exceptional ways for presenting the discovered knowledge to facilitate the development of treatment and management plans for patients and their caregivers. Overall, dementia assessment tools that utilize Al techniques better performance when making diagnostic-related decisions since they rely on models derived from processing historical cases and controls rather using conventional methods like scoring functions. This makes the diagnosis process simple, accurate, and convenient for clinicians and medical professionals.

Out of all considered dementia screening apps, Cognity utilizes Al for dementia screening; this app analyses a photo of a clock drawing done by the user and then, based a large sample of patients, determines the probability of dementia risk. The ACE app also has an inbuilt mechanism for automated administration, scoring, and reporting using the human factor approach. Findings of Newman et al., (2018) suggest that the computerized version (ACE app) can capture measures more accurately than the original version of the ACE medical assessment. MOBI-COG, the digitalized version of Mini-Cog test, is also a fully automated mobile app that uses $M L$ techniques to recognize hand-written digits and characters. It uses a k-nearest neighbour (kNN) algorithm (Gonzalez \& Woods, 2002) to identify the characteristics of the clock drawing done by the suspected individual.

It's clear that integrating technological advancements into traditional dementia screening assessments is a topic that has not yet gained enough attention from the relevant research bodies. Therefore, more research that focuses on improving the accuracy and efficiency of the existing dementia screening tools using advanced intelligent technology is required.

\section{Conclusions}

Dementia is a type of cognitive dysfunction that is common among elderly people. Screening for dementia is the key for successful intervention, therefore regular check-ups are critical to identify this problem at an early stage. This paper highlights and critically analyzes mobile-based technology tools for dementia screening in terms of various criteria: accessibility, comprehensiveness, performance, efficiency, and usability among others. A critical analysis was conducted to identify all the prevailing dementia screening apps along with their prominent features. According to the results of the evaluation, the DRT app is identified as one of the most rated apps with a rating of $4.8 / 5$ and over 1000 downloads. The Dementia Screener is the app that gained the lowest ratings although it had over 1000 recorded downloads. Apps such as MoCA, BCl, ALZ, DST, and Cognitive Exams cover more neurocognitive areas based on DSM-5 criteria; the Brain Test, ACE Mobile, CDD, BrainCheck, Cognity, and 6CIT apps are validated to be used in clinical setups. However, all the dementia screening apps have their own pros and cons; some apps are comprehensive, but time-consuming, whereas others are efficient and comprehensive, but have limited accessibility. The ACE dementia screening app is comprehensive, efficient, and available freely on both Android and iOS platforms, but access is limited to healthcare professionals only. Therefore, the need for a dementia screening app that meets the neurocognitive domains defined in the DSM-5 and that is accessible, is essential. Further research and development should be carried out to design and implement a more accurate and reliable screening tool to facilitate early diagnosis of dementia traits. This can be accomplished by using new technology such as Al and machine learning in which the detection of dementia traits will be solely based on former instances (cases and controls) rather than simple scores generated by conventional medical assessment tools such as the ones we surveyed.

\section{References}

Abbott, R., White, L., Ross, G., Masaki, K., Curb, J., \& Petrovitch, H. (2004). Walking and dementia in physically capable elderly men. American Medical Association, 1447-1454. 
Alzheimers Association. (2019). New diagnostic criteria and guidelines for Alzheimer's disease. Retrieved from Alzheimer's Association: https://www.alz.org/research

Apple Inc. (2019a). 6CIT. Retrieved from https://apps.apple.com/us/app/6cit/id1442965681

Apple Inc. (2019b). Dementia test - risk calculator of dementia. Retrieved from https://apps.apple.com/us/app/ dementia-test-risk-calculator-of-dementia/id1014958634

Apple Inc. (2019c). MoCA App. Retrieved from https://apps.apple.com/us/app/moca-app/id1206246590

Aske, D. (1990). The correlation between mini-mental state examination scores and Katz ADL status among dementia patients. Rehabilitation Nursing, 140-146.

Baadel S., Thabtah F. Lu J. (2020) A Clustering Approach for Autism based Autistic Trait Classification. Informatic for Health and Social Care Journal. 2020 Feb 3:1-18. doi: 10.1080/17538157.2019.1687482.

Berauk, V., Murugiah, M., Soh, Y., Sheng, Y., Wong, T., \& Ming, L. (2017). Mobile health applications for caring of older people: Review and comparison. Therapeutic Innovation \& Regulatory Science, 1-9.

Borson, S., Scanlan, J., Brush, M., Vitaliano, P., \& Dokmak, A. (2000). The Mini-Cog: a cognitive 'vital signs' measure for dementia screening in multi-lingual elderly. International Journal of Geriatric Psychiatry, 15(11), , 1021-1027.

BrainCheck. (2019). About us. Retrieved from https://braincheck.com/about

BrainTest Inc. (2013). Take the brain test. Retrieved from https://braintest.com/

Brodaty, H., Pond, D., Kemp, N., Luscombe, G., Harding, L., Berman, K., \& Huppert, F. (2002). The GPCOG: A new screening test for dementia designed for general practice. Journal of the American Geriatric Society. DOI: 10.1046/j.1532-5415.2002.50122.x.

Bruno, D., \& Vignaga, S. (2019). Addenbrooke's cognitive examination III in the diagnosis of dementia: A critical review. Neuropsychiatric Disease and Treatment, 441-447.

Buschke, H., Kuslansky, G., Katz, M., Stewart, W., Sliwinski, M., Eckholdt, H., \& Lipton, R. (1999). Screening for dementia with the Memory Impairment Screen. Neurology. doi.org/10.1212/WNL.52.2.231.

Butcher, J. (2007). CAIDE dementia risk score validated in study. Clinical Neurology News, 22a-22b.

Caramelli, P., Carthery-Goulart, M., Porto, C., Charchat-Fichman, H., \& Nitrini, R. (2007). Category fluency as a screening test for Alzheimer disease in illiterate and literate patients. Alzheimer Disease \& Associated Disorders, 65-67.

Callahan, C., Unverzagt, F., Hui, S., Perkins, A., \& Hendrie, H. (2002). Six-item screener to identify cognitive impairment among potential subjects for clinical research. Indiana: Lippincott Williams \& Wilkins.

Chertkow, H. (2008). Introducing a series based on the third Canadian consensus conference on the diagnosis and treatment of dementiua. Canadian Medical Association, 178(3), 316-321

ChewyLogic (2019). A digital version of St Louis University Mental Status Exam (SLUMS). Retrieved from https://www.eslumstest.com/

Cicerone, K., \& Azulay, J. (2002). Diagnostic utility of attention measures in postconcussion syndrome. Journal the Clinical Neuropsychologist, 280-289. Retrieved from https://doi.org/10.1076/clin.16.3.280.13849

Comerford, V. E., Geffen, G. M., May, C., Medland , S., \& Geffen, L. (2002). A rapid screen of the severity of mild traumatic brain injury. Journal of Clinical and Experimental Neuropsychology, 409-419. Retrieved from https://doi.org/10.1076/jcen.24.4.409.1044 
Cordella, C., Borson, S., Boustan, M., Chodosh, J., Reuben, D., Verghese, J., . . Fried, L. (2013). Alzheimer's Association recommendations for operationalizing the detection of cognitive impairment during the Medicare annual wellness visit in a primary care setting. Alzheimer's \& Dementia 9, 141-150.

Ehrensperger, M., Taylor, K., Berres, M., Foldi, N., Dellenbach, M., Bopp, I., . . Monsch, A. (2014). BrainCheck A very brief tool to detect incipient cognitive decline: Optimized case-finding combining patient- and informant-based data. Alzheimer's Research \& Therapy, DOI: 10.1186/s13195-014-0069-y.

Ernst, R., \& Hay, J. (1999). The U.S. economic and social costs of Alzheimer's disease revisited. American Journal of Public Health, 1261-1264

Fan, J., Flombaumb, J., McCandliss, B., Thomas, K., \& Posner, M. ( 2003). Cognitive and brain consequences of conflict. Neurolmage, 42-57.

Folstein, M., Folstein , S., \& McHugh, P. (1975). Mini-mental state: a practical method for grading the cognitive state of patients for the clinician. Journal of Psychiatric, 12 (3), 189-198,

Flaherty, L., Midden, A., \& Mast, B. (2019). Psychometric evaluation of the symptoms of dementia screener (SDS) in a geriatric primary care population. Journal Clinical Gerontologist, 504-511. doi:DOI: 10.1080/07317115.2018.1453906

Friedman, M., Leach, L., Kaplan, E., Winocur, G., Shulman, K., \& Delis, D. (1994). DC: Clock drawing: A neuropsychological analysis. New York: Oxford University Press.

Galvin, J., Roe, C., Powlishta K., Coats, M., Muich S., Grant E., . . . Morris, J. (2005 ). The AD8: A brief informant interview to detect dementia. Neurology, 559-64.

Gonzalez, R., \& Woods, R. (2002). Digital image processing, 2nd edition. Upper Saddle River, New Jersey: Prentice Hall.

Google. (2016). MMSE. Retrieved from https://play.google.com/store/apps/details? id=com.yasintanriverdi.mmse

Google. (2017). Dementia \& Alzheimer's memory diagnosis test: MMSE. Retrieved from https://play.google.com/store/apps/details?id=com.alzheimers_mme

Google. (2019a). Cognitive exams. Retrieved fromhttps://play.google.com/store/apps/details? id=br.com.digos.examescognitivos

Google. (2019b). Dementia risk tool. Retrieved from https://play.google.com/store/apps/details? id=com.dementiarisktool

Google. (2019c). DST - Dementia screening test, Alzheimer test. Retrieved from https://play.google.com/store/ apps/details?id=com.dementiascreeningtest

Greenwald, B., Cifu, D., Marwitz, J., Enders, L., Brown, A., Englander, J., \& Zafonte, R. (2001). Factors associated with balance deficits on admission to rehabilitation after traumatic brain injury: A multicenter analysis. Journal of Head Trauma Rehabilitation, 238-252.

Hodges, J., \& Larner, A. (2017). Addenbrooke's cognitive examinations: ACE, ACE-R, ACE-III, ACEapp, and MACE. Cognitive Screening Instruments, 109-137.

Holsinger, T., Deveau, J., Boustani, M., \& Williams, J. (2007). Does this patient have dementia?. Journal of American Medical Association, 297: 2391-2404. DOI 10.1001/jama.297.21.2391.

Hodkinson, H. (1972). Evaluation of a mental test score for assessment of mental impairment in the elderly. Age and Aging, 233-238. 
Inouye, S., Dyck, C., Alessi, C., Balkin, S., Siegal, A., \& Horwitz, R. (1990). Clarifying confusion: The confusion assessment method - A new method for the detection of delirium. Journal of Annals of Internal Medicine, 941-948.

Inoven. (2018). Cognity. Retrieved from https://cognity.app

International Telecommunication Union. (2015). Measuring the information society report. Geneva: International Telecommunication Union.

Jorm, A., Scott, R., \& Jacomb, P. (1989). Assessment of cognitive decline in dementia by informant questionnaire. International Journal of Geriatric Psychiatry. Retrieved from https://doi.org/10.1002/gps.930040109.

Johnson, L., Cushing, B., Rohlfing, G., Edwards, M., Davenloo, H., D'Agostino, D., . . O'Bryant, S. (2014). The Hachinski Ischemic scale and cognition: The influence of ethnicity. Age and Ageing, 43,(3), 364-369.

Julayanont, P., Tangwongchai, S., Hemrungrojn, S., Tunvirachaisakul, C., Phanthumchinda, K., Hongsawat, J., . . . Nasreddine, Z. (2015). The Montreal cognitive assessment-Basic: A screening tool for mild cognitive impairment in illiterate and low-educated elderly adults. Journal of the American Geriatrics Society, 63(12), 2550-2554.

Kansagara, D., \& Freeman, M. (2010). A systematic evidence review of the signs and symptoms of dementia and brief cognitive tests available in VA [Internet]. Washington (DC): Department of Veterans Affairs (US).

Kivipelto, M., Ngandu, T., Laatikainen, T., Winblad, B., Soininen, H., \& Tuomilehto, J. (2006). Risk score for the prediction of dementia risk in 20 years among middle aged people: A longitudinal, population-based study. The Lancent Neurology, 735-741.

Klimova, B. (2017). Mobile phone apps in the management and assessment of mild cognitive impairment and/ or mild-to-moderate ementia: An opinion article on recent findings. Frontiers in Human Neuroscience. DOI: 10.3389/fnhum.2017.00461

Market Research. (2015). Healthcare mobility solutions market by products \& services (mobile devices, mobile apps, enterprise platforms), application (patient care, operations, workforce management), end user (payers, providers, patients) - Global forecast to 2020. Dallas, TX: Markets and Markets.

Melara,, R., \& Algom, D. (2003). Driven by information: A tectonic theory of Stroop effects. Psychological Review, 110(3), , 422-471. Retrieved from https://doi.org/10.1037/0033-295X.110.3.422.

Monte, V., Geffen , G., May, C., \& McFarland, K. (2010). Improved sensitivity of the rapid screen of mild traumatic brain injury. Journal of Clinical and Experimental Neuropsychology, 28-37.

Mundt, J. C., Freed, D. M., \& Greist, J. H. (2000). Lay person-based screening for early detection of Alzheimer's disease: Development and validation of an instrument. The Journals of Gerontology: Series B: Psychological Sciences and Social Sciences, 55(3), P163-P170

Nasreddine, Z., Phillips, N., Bédirian, V., Charbonneau, S., Whitehead, V., Collin, I., . . Chertkow, H. (2005). The Montreal cognitive assessment, MoCA: A brief screening tool for mild cognitive impairment. Journal of the American Geriatrics Society, 695-699.

Newman, C., Bevin, A., Zajicek, J., Hodges, J., Vuillermoz, E., Dickenson, J., . . . Noad, R. (2018). Improving the quality of cognitive screening assessments: ACEmobile, an iPad-based version of the Addenbrooke's cognitive examination-III. Alzheimer's \& Dementia: Diagnosis, Assessment \& Disease Monitoring, 182187.

Nirjon, S., Emi, I. A., Sayeed Mondol, M. A., Salekin, A., \& Stankovic, J. (2014). MOBI-COG: A mobile application for instant screening of dementia using the mini-cog test. 5th Conference on Wireless Health. Bethesda, United States: Association for Computing Machinery, Inc. 
Noone, P. (2015). Addenbrooke's cognitive examination-III. Occupational Medicine, 65(5), 418-420.

Ouimette, P. \& Klein, D. (1995). est-Retest stability, mood-state dependence, and informant-subject concordance of the SCID-AXIS II questionnaire in a non-clinical sample. Journal of Personality Disorders, 105-111.

Panegyres, P., Berry, R., \& Burchell, J. (2016). Early dementia screening. Diagnostics (Basel),DOI: 10.3390/diagnostics6010006.

Sangha, S., George, J., Winthrop, C., \& Panchal, S. (2015). Confusion: Delirium and dementia - a smartphone app to improve cognitive assessment. [Report]. BMJ. DOI: 10.1136/bmjquality.u202580.w1592.

Scharre, D., Chang, S.-I., Murden, R., Lamb, J., Beversdorf, D., Kataki, M., . . . Bornstein, R. (2010). Selfadministered gerocognitive examination (SAGE): A brief cognitive assessment instrument for mild cognitive impairment $(\mathrm{MCl})$ and early dementia. Journal of Alzheimer Disease and Associated Disorders, 64-71.

Scharre, D., Chang, S., Nagaraja, H., Vrettos, N., \& Bornstein, R. (2017). Digitally translated self-administered gerocognitive examination (eSAGE): Relationship with its validated paper version, neuropsychological evaluations, and clinical assessments. Alzheimer's Research \& Therapy, 9(44). DOI:10.1186/s13195017-0269-3

Sindi, S., Calov, E., Fokkens, J., Ngandu, T., Soininen, H., Tuomilehto, J., \& Kivipelto, M. (2015). The CAIDE dementia risk score app: The development of an evidence-based mobile application to predict the risk of dementia. Alzheimer's \& Dementia: Diagnosis, Assessment \& Disease Monitoring, 328-333.

Snyderman, D., \& Rovner, B. (2009). Mental status examination in primary care: A review. American Family Physician, 809-814.

Statista. (2018). Global mobile OS market share 2009-2018, by quarter. Retrieved fromhttps://www.statista.com/statistics/266136/global-market-share-held-by-smartphoneoperating-systems/

Tappen, R., Rosselli, M., \& Engstrom, G. (2009). Evaluation of the functional activities questionnaire (FAQ) in cognitive screening across four American ethnic groups. Journal of the Clinical Neuropsychologist, 646-661.

Tariq, S., Tumosa, N., Chibnall, J., Perry, M., \& Morley, J. (2006). Comparison of the Saint Louis University mental status examination and the mini-mental state examination for detecting dementia and mild neurocognitive disorder-a pilot study. The American Journal of Geriatric Psychiatry, 14(11), 900-910.

Thabtah, F. (2019). Machine learning in autistic spectrum disorder behavioral research: A review and ways forward. Informatics for Health and Social Care, 278-297.

Thabtah F, Abdelhamid N, Peebles D (2019) A machine learning autism classification based on logistic regression analysis. Health information science and systems 7 (1), 12.

Thabtah F., Mampusti E., Peebles P., Herradura R, Varghese J. (2020) A Mobile-Based Screening System for Data Analyses of Early Dementia Traits Detection. Journal Medical Systems (2020) 44: 24. https://doi.org/10.1007/s10916-019-1469-0.

Thabtah F. (2019) An Accessible and Efficient Autism Screening Method for Behavioural Data and Predictive Analyses. Health Informatics Journal. 19:1460458218796636. doi: 10.1177/1460458218796636. 2018.

Torre, J. (2004). Is Alzheimer's disease a neurodegenerative or a vascular disorder? Data, dogma, and dialectics. The Lancet Neurology, 3(3), 184-190. DOI:10.1016/s1474-4422(04)00683-0

Ventola, C. (2014). Mobile devices and apps for health care professionals: Uses and benefits. Pharmacy \& Therapeutics, 356-364. 
Wallace, S., Clark, M., \& White, J. (2012). It's on my iPhone': Attitudes to the use of mobile computing devices in medical education, a mixed-methods study. BJM Open, 23-56.

Yang, S., Flores, B., Magal, R., Harris, K., Gross, J., Ewbank, A., . . Eagleman, D. (2017). Diagnostic accuracy of tablet-based software for the detection of concussion. PLOS ONE 12. Retrieved from https://doi.org/10.1371/journal.pone.0179352. 
Yesavage, J., Brink, T., Rose, T., Lum, O., Huang, V., Adey, M., \& Leirer, V. (1982). Development and validation of a geriatric depression screening scale: A preliminary report. Journal of Psychiatric Research, 37-49.

Yoganathan, S. (2013). Review of the iPhone app for delirium and dementia. Retrieved from https://britishgeriatricssociety.wordpress.com/2013/12/27/review-of-the-iphone-app-for-deliriumand-dementia/ 